\title{
Ostrowski's Type Inequalities for the Complex Integral on Paths
}

\section{SILVESTRU SEVER DRAGOMIR*}

ABSTRACT. In this paper, we extend the Ostrowski inequality to the integral with respect to arc-length by providing upper bounds for the quantity

$$
\left|f(v) \ell(\gamma)-\int_{\gamma} f(z)\right| d z||
$$

under the assumptions that $\gamma$ is a smooth path parametrized by $z(t), t \in[a, b]$ with the length $\ell(\gamma), u=z(a)$, $v=z(x)$ with $x \in(a, b)$ and $w=z(b)$ while $f$ is holomorphic in $G$, an open domain and $\gamma \subset G$. An application for circular paths is also given.

Keywords: Complex integral, continuous functions, holomorphic functions, Ostrowski inequality.

2020 Mathematics Subject Classification: 26D15, 26D10, 30A10, 30A86.

\section{INTRODUCTION}

In 1938, A. Ostrowski [8], proved the following inequality concerning the distance between the integral mean $\frac{1}{b-a} \int_{a}^{b} f(t) d t$ and the value $f(x), x \in[a, b]$.

Theorem 1.1 (Ostrowski, 1938 [8]). Let $f:[a, b] \rightarrow \mathbb{R}$ be continuous on $[a, b]$ and differentiable on $(a, b)$ such that $f^{\prime}:(a, b) \rightarrow \mathbb{R}$ is bounded on $(a, b)$, i.e., $\left\|f^{\prime}\right\|_{\infty}:=\sup _{t \in(a, b)}\left|f^{\prime}(t)\right|<\infty$. Then,

$$
\left|f(x)-\frac{1}{b-a} \int_{a}^{b} f(t) d t\right| \leq\left[\frac{1}{4}+\left(\frac{x-\frac{a+b}{2}}{b-a}\right)^{2}\right]\left\|f^{\prime}\right\|_{\infty}(b-a)
$$

for all $x \in[a, b]$ and the constant $\frac{1}{4}$ is the best possible.

In [6], S. S. Dragomir and S. Wang, by the use of the Montgomery integral identity [7, p. 565],

$$
f(x)-\frac{1}{b-a} \int_{a}^{b} f(t) d t=\frac{1}{b-a} \int_{a}^{b} p(x, t) f^{\prime}(t) d t, \quad x \in[a, b],
$$

where $p:[a, b]^{2} \rightarrow \mathbb{R}$ is given by

$$
p(x, t):=\left\{\begin{array}{lll}
t-a & \text { if } & t \in[a, x] \\
t-b & \text { if } & t \in(x, b]
\end{array}\right.
$$

Received: 23.09.2020; Accepted: 02.11.2020; Published Online: 03.11.2020

*Corresponding author: Silvestru Sever Dragomir; sever.dragomir@vu.edu.au

DOI:10.33205/cma.798861 
gave a simple proof of Ostrowski's inequality and applied it for special means (identric mean, logarithmic mean, etc.) and to the problem of estimating the error bound in approximating the Riemann integral $\int_{a}^{b} f(t) d t$ by one arbitrary Riemann sum (see [6, Section 3]). For extensions of Ostrowski's inequality in terms of the $p$-norms of the derivative, see [1], [2] and [3]. For a recent survey on Ostrowski's inequality, see [4].

In order to extend this result for the complex integral, we need some preparations as follows. Suppose $\gamma$ is a smooth path parametrized by $z(t), t \in[a, b]$ and $f$ is a complex function which is continuous on $\gamma$. Put $z(a)=u$ and $z(b)=w$ with $u, w \in \mathbb{C}$. We define the integral of $f$ on $\gamma_{u, w}=\gamma$ as

$$
\int_{\gamma} f(z) d z=\int_{\gamma_{u, w}} f(z) d z:=\int_{a}^{b} f(z(t)) z^{\prime}(t) d t .
$$

We observe that the actual choice of parametrization of $\gamma$ does not matter. This definition immediately extends to paths that are piecewise smooth. Suppose $\gamma$ is parametrized by $z(t)$, $t \in[a, b]$, which is differentiable on the intervals $[a, c]$ and $[c, b]$, then assuming that $f$ is continuous on $\gamma$ we define

$$
\int_{\gamma_{u, w}} f(z) d z:=\int_{\gamma_{u, v}} f(z) d z+\int_{\gamma_{v, w}} f(z) d z
$$

where $v:=z(c)$. This can be extended for a finite number of intervals. We also define the integral with respect to arc-length

$$
\int_{\gamma_{u, w}} f(z)|d z|:=\int_{a}^{b} f(z(t))\left|z^{\prime}(t)\right| d t
$$

and the length of the curve $\gamma$ is then

$$
\ell(\gamma)=\int_{\gamma_{u, w}}|d z|=\int_{a}^{b}\left|z^{\prime}(t)\right| d t
$$

Let $f$ and $g$ be holomorphic in $G$, an open domain and suppose $\gamma \subset G$ is a piecewise smooth path from $z(a)=u$ to $z(b)=w$. Then, we have the integration by parts formula

$$
\int_{\gamma_{u, w}} f(z) g^{\prime}(z) d z=f(w) g(w)-f(u) g(u)-\int_{\gamma_{u, w}} f^{\prime}(z) g(z) d z .
$$

We recall also the triangle inequality for the complex integral, namely

$$
\left|\int_{\gamma} f(z) d z\right| \leq \int_{\gamma}|f(z)||d z| \leq\|f\|_{\gamma, \infty} \ell(\gamma),
$$

where $\|f\|_{\gamma, \infty}:=\sup _{z \in \gamma}|f(z)|$.

We also define the $p$-norm with $p \geq 1$ by

$$
\|f\|_{\gamma, p}:=\left(\int_{\gamma}|f(z)|^{p}|d z|\right)^{1 / p} .
$$

For $p=1$, we have

$$
\|f\|_{\gamma, 1}:=\int_{\gamma}|f(z)||d z| .
$$

If $p, q>1$ with $\frac{1}{p}+\frac{1}{q}=1$, then by Hölder's inequality, we have

$$
\|f\|_{\gamma, 1} \leq[\ell(\gamma)]^{1 / q}\|f\|_{\gamma, p} .
$$

In the recent paper [5], we obtained the following result for functions of complex variable: 
Theorem 1.2. Let $f$ be holomorphic in $G$, an open domain and suppose $\gamma \subset G$ is a smooth path from $z(a)=u$ to $z(b)=w$. If $v=z(x)$ with $x \in(a, b)$, then $\gamma_{u, w}=\gamma_{u, v} \cup \gamma_{v, w}$,

$$
\begin{aligned}
& \left|f(v)(w-u)-\int_{\gamma} f(z) d z\right| \\
\leq & \left\|f^{\prime}\right\|_{\gamma_{u, v} ; \infty} \int_{\gamma_{u, v}}|z-u||d z|+\left\|f^{\prime}\right\|_{\gamma_{v, w} ; \infty} \int_{\gamma_{v, w}}|z-w||d z| \\
\leq & {\left[\int_{\gamma_{u, v}}|z-u||d z|+\int_{\gamma_{v, w}}|z-w||d z|\right]\left\|f^{\prime}\right\|_{\gamma_{u, w} ; \infty} }
\end{aligned}
$$

and

$$
\begin{aligned}
& \left|f(v)(w-u)-\int_{\gamma} f(z) d z\right| \\
\leq & \max _{z \in \gamma_{u, v}}|z-u|\left\|f^{\prime}\right\|_{\gamma_{u, v} ; 1}+\max _{z \in \gamma_{v, w}}|z-w|\left\|f^{\prime}\right\|_{\gamma_{v, w} ; 1} \\
\leq & \max \left\{\max _{z \in \gamma_{u, v}}|z-u|, \max _{z \in \gamma_{v, w}}|z-w|\right\}\left\|f^{\prime}\right\|_{\gamma_{u, w} ; 1} .
\end{aligned}
$$

If $p, q>1$ with $\frac{1}{p}+\frac{1}{q}=1$, then

$$
\begin{aligned}
& \left|f(v)(w-u)-\int_{\gamma} f(z) d z\right| \\
\leq & \left(\int_{\gamma_{u, v}}|z-u|^{q}|d z|\right)^{1 / q}\left\|f^{\prime}\right\|_{\gamma_{u, v} ; p}+\left(\int_{\gamma_{v, w}}|z-w|^{q}|d z|\right)^{1 / q}\left\|f^{\prime}\right\|_{\gamma_{v, w} ; p} \\
\leq & \left(\int_{\gamma_{u, v}}|z-u|^{q}|d z|+\int_{\gamma_{v, w}}|z-w|^{q}|d z|\right)^{1 / q}\left\|f^{\prime}\right\|_{\gamma_{u, w} ; p} .
\end{aligned}
$$

Motivated by the above results, in this paper, we extend the Ostrowski inequality to the complex integral, by providing upper bounds for the quantity

$$
\left|f(v) \ell(\gamma)-\int_{\gamma} f(z)\right| d z||
$$

under the assumptions that $\gamma$ is a smooth path parametrized by $z(t), t \in[a, b]$, with the length $\ell(\gamma), u=z(a), v=z(x)$ with $x \in(a, b)$ and $w=z(b)$ while $f$ is holomorphic in $G$, an open domain and $\gamma \subset G$. An application for circular paths is also given.

\section{Ostrowski Type Results}

We have the following result for functions of complex variable: 
Theorem 2.3. Let $f$ be holomorphic in $G$, an open domain and suppose $\gamma \subset G$ is a smooth path from $z(a)=u$ to $z(b)=w$. If $v=z(x)$ with $x \in(a, b)$, then $\gamma_{u, w}=\gamma_{u, v} \cup \gamma_{v, w}$ and

$$
\begin{aligned}
\left|f(v) \ell(\gamma)-\int_{\gamma} f(z)\right| d z|| \leq & \ell\left(\gamma_{u, v}\right)\left\|f^{\prime}\right\|_{\gamma_{u, v} ; 1}+\ell\left(\gamma_{v, w}\right)\left\|f^{\prime}\right\|_{\gamma_{v, w} ; 1} \\
\leq & \left\{\begin{array}{l}
\frac{1}{2}\left[\ell\left(\gamma_{u, w}\right)+\left|\ell\left(\gamma_{u, v}\right)-\ell\left(\gamma_{v, w}\right)\right|\right]\left\|f^{\prime}\right\|_{\gamma_{u, w} ; 1}, \\
{\left[\ell^{p}\left(\gamma_{u, v}\right)+\ell^{p}\left(\gamma_{v, w}\right)\right]^{1 / p}\left(\left\|f^{\prime}\right\|_{\gamma_{u, v} ; 1}^{q}+\left\|f^{\prime}\right\|_{\gamma_{v, w} ; 1}^{q}\right)^{1 / q}} \\
p, q>1 \text { and } \frac{1}{p}+\frac{1}{q}=1, \\
\frac{1}{2} \ell\left(\gamma_{u, w}\right)\left[\left\|f^{\prime}\right\|_{\gamma_{u, w} ; 1}+\left|\left\|f^{\prime}\right\|_{\gamma_{u, v} ; 1}-\left\|f^{\prime}\right\|_{\gamma_{v, w} ; 1}\right|\right] .
\end{array}\right.
\end{aligned}
$$

Proof. Using the integration by parts formula, we have

$$
\begin{aligned}
\int_{\gamma_{u, v}} f(z)|d z| & =\int_{a}^{x} f(z(t))\left|z^{\prime}(t)\right| d t \\
& =\int_{a}^{x} f(z(t)) d\left(\int_{a}^{t}\left|z^{\prime}(s)\right| d s\right) \\
& =\left.f(z(t)) \int_{a}^{t}\left|z^{\prime}(s)\right| d s\right|_{a} ^{x}-\int_{a}^{x} \frac{d f(z(t))}{d t}\left(\int_{a}^{t}\left|z^{\prime}(s)\right| d s\right) d t \\
& =f(z(x)) \int_{a}^{x}\left|z^{\prime}(s)\right| d s-\int_{a}^{x} f^{\prime}(z(t))\left(\int_{a}^{t}\left|z^{\prime}(s)\right| d s\right) z^{\prime}(t) d t \\
& =f(v) \ell\left(\gamma_{u, v}\right)-\int_{a}^{x} f^{\prime}(z(t))\left(\int_{a}^{t}\left|z^{\prime}(s)\right| d s\right) z^{\prime}(t) d t
\end{aligned}
$$

and

$$
\begin{aligned}
\int_{\gamma_{v, w}} f(z)|d z| & =\int_{x}^{b} f(z(t))\left|z^{\prime}(t)\right| d t \\
& =-\int_{x}^{b} f(z(t)) d\left(\int_{t}^{b}\left|z^{\prime}(s)\right| d s\right) \\
& =-\left.f(z(t)) \int_{t}^{b}\left|z^{\prime}(s)\right| d s\right|_{x} ^{b}+\int_{x}^{b} \frac{d f(z(t))}{d t}\left(\int_{t}^{b}\left|z^{\prime}(s)\right| d s\right) d t \\
& =f(z(x)) \int_{x}^{b}\left|z^{\prime}(s)\right| d s+\int_{x}^{b} f^{\prime}(z(t))\left(\int_{t}^{b}\left|z^{\prime}(s)\right| d s\right) z^{\prime}(t) d t \\
& =f(v) \ell\left(\gamma_{v, w}\right)+\int_{x}^{b} f^{\prime}(z(t))\left(\int_{t}^{b}\left|z^{\prime}(s)\right| d s\right) z^{\prime}(t) d t .
\end{aligned}
$$


If we add these two equalities, we get

$$
\begin{aligned}
& \int_{\gamma_{u, v}} f(z)|d z|+\int_{\gamma_{v, w}} f(z)|d z| \\
= & f(v) \ell\left(\gamma_{u, v}\right)+f(v) \ell\left(\gamma_{v, w}\right)-\int_{a}^{x} f^{\prime}(z(t))\left(\int_{a}^{t}\left|z^{\prime}(s)\right| d s\right) z^{\prime}(t) d t \\
+ & \int_{x}^{b} f^{\prime}(z(t))\left(\int_{t}^{b}\left|z^{\prime}(s)\right| d s\right) z^{\prime}(t) d t,
\end{aligned}
$$

which gives the following equality of interest

$$
\begin{aligned}
& f(v) \ell\left(\gamma_{u, w}\right)-\int_{\gamma_{u, w}} f(z)|d z| \\
= & \int_{a}^{x} f^{\prime}(z(t))\left(\int_{a}^{t}\left|z^{\prime}(s)\right| d s\right) z^{\prime}(t) d t-\int_{x}^{b} f^{\prime}(z(t))\left(\int_{t}^{b}\left|z^{\prime}(s)\right| d s\right) z^{\prime}(t) d t .
\end{aligned}
$$

By taking the modulus in (2.9) and using the properties of modulus, we get

$$
\begin{aligned}
& \left|f(v) \ell\left(\gamma_{u, w}\right)-\int_{\gamma_{u, w}} f(z)\right| d z|| \\
\leq & \left|\int_{a}^{x} f^{\prime}(z(t))\left(\int_{a}^{t}\left|z^{\prime}(s)\right| d s\right) z^{\prime}(t) d t\right| \\
+ & \left|\int_{x}^{b} f^{\prime}(z(t))\left(\int_{t}^{b}\left|z^{\prime}(s)\right| d s\right) z^{\prime}(t) d t\right| \\
\leq & \int_{a}^{x}\left|f^{\prime}(z(t))\right|\left|z^{\prime}(t)\right|\left(\int_{a}^{t}\left|z^{\prime}(s)\right| d s\right) d t \\
& +\int_{x}^{b}\left|f^{\prime}(z(t))\right|\left|z^{\prime}(t)\right|\left(\int_{t}^{b}\left|z^{\prime}(s)\right| d s\right) d t=: B(x)
\end{aligned}
$$

for $x \in[a, b]$. We have

$$
\int_{a}^{t}\left|z^{\prime}(s)\right| d s \leq \int_{a}^{x}\left|z^{\prime}(s)\right| d s \text { for } t \in[a, x]
$$

and

$$
\int_{t}^{b}\left|z^{\prime}(s)\right| d s \leq \int_{x}^{b}\left|z^{\prime}(s)\right| d s \text { for } t \in[x, b],
$$

then

$$
\begin{aligned}
B(x) & \leq \int_{a}^{x}\left|z^{\prime}(s)\right| d s \int_{a}^{x}\left|f^{\prime}(z(t))\right|\left|z^{\prime}(t)\right| d t \\
& +\int_{x}^{b}\left|z^{\prime}(s)\right| d s \int_{x}^{b}\left|f^{\prime}(z(t))\right|\left|z^{\prime}(t)\right| d t \\
& =\ell\left(\gamma_{u, v}\right) \int_{\gamma_{u, v}}\left|f^{\prime}(z)\right||d z|+\ell\left(\gamma_{v, w}\right) \int_{\gamma_{v, w}}\left|f^{\prime}(z)\right||d z|
\end{aligned}
$$


and by (2.10), we get the first inequality in (2.8). The second part follows by Hölder's inequalities

$$
m n+c d \leq\left\{\begin{array}{l}
\max \{m, c\}(n+d) \\
\left(m^{p}+c^{p}\right)^{1 / p}\left(n^{q}+d^{q}\right)^{1 / q}, \text { for } p, q>1, \frac{1}{p}+\frac{1}{q}=1,
\end{array}\right.
$$

where $m, n, c, d \geq 0$.

Corollary 2.1. With the assumption of Theorem 2.3 and if there exists $m \in \gamma$ such that $\ell\left(\gamma_{u, m}\right)=$ $\ell\left(\gamma_{m, w}\right)$, then

$$
\left|f(m) \ell(\gamma)-\int_{\gamma} f(z)\right| d z|| \leq \frac{1}{2} \ell(\gamma)\left\|f^{\prime}\right\|_{\gamma_{u, w} ; 1}
$$

and if $s \in \gamma$ such that $\int_{\gamma_{u, s}}\left|f^{\prime}(z)\right||d z|=\int_{\gamma_{s, w}}\left|f^{\prime}(z)\right||d z|$, then

$$
\left|f(s) \ell(\gamma)-\int_{\gamma} f(z)\right| d z|| \leq \frac{1}{2} \ell(\gamma)\left\|f^{\prime}\right\|_{\gamma_{u, w} ; 1} .
$$

We have also the following result for $p$-norms:

Theorem 2.4. With the assumption of Theorem 2.3, we have for $p, q>1$ with $\frac{1}{p}+\frac{1}{q}=1$ that

$$
\begin{aligned}
& \quad\left|f(v) \ell(\gamma)-\int_{\gamma} f(z)\right| d z|| \\
& \leq \frac{1}{q+1}\left[\ell^{1+1 / q}\left(\gamma_{u, v}\right)\left\|f^{\prime}\right\|_{\gamma_{u, v} ; p}+\ell^{1+1 / q}\left(\gamma_{v, w}\right)\left\|f^{\prime}\right\|_{\gamma_{v, w} ; p}\right] \\
& \leq \frac{1}{q+1}\left\{\begin{array}{l}
\frac{1}{2^{1+1 / q}}\left[\ell\left(\gamma_{u, w}\right)+\left|\ell\left(\gamma_{u, v}\right)-\ell\left(\gamma_{v, w}\right)\right|\right]^{1+1 / q} \\
\times\left[\left\|f^{\prime}\right\|_{\gamma_{u, v} ; p}+\left\|f^{\prime}\right\|_{\gamma_{v, w} ; p}\right], \\
{\left[\begin{array}{l}
\left.\ell^{q+1}\left(\gamma_{u, v}\right)+\ell^{q+1}\left(\gamma_{v, w}\right)\right]^{1 / q}\left\|f^{\prime}\right\|_{\gamma_{u, w} ; p}, \\
\frac{1}{2^{1 / p}}\left[\left\|f^{\prime}\right\|_{\gamma_{u, w} ; p}^{p}+\left|\left\|f^{\prime}\right\|_{\gamma_{u, v} ; p}^{p}-\left\|f^{\prime}\right\|_{\gamma_{v, w} ; p}^{p}\right|\right]^{1 / p} \\
\times\left[\ell^{1+1 / q}\left(\gamma_{u, v}\right)+\ell^{1+1 / q}\left(\gamma_{v, w}\right)\right] .
\end{array}\right.}
\end{array}\right.
\end{aligned}
$$

Proof. Using the weighted Hölder integral inequality for $p, q>1$ with $\frac{1}{p}+\frac{1}{q}=1$, we have

$$
\begin{aligned}
& \int_{a}^{x}\left|f^{\prime}(z(t))\right|\left|z^{\prime}(t)\right|\left(\int_{a}^{t}\left|z^{\prime}(s)\right| d s\right) d t \\
\leq & \left(\int_{a}^{x}\left|f^{\prime}(z(t))\right|^{p}\left|z^{\prime}(t)\right| d t\right)^{1 / p}\left(\int_{a}^{x}\left(\int_{a}^{t}\left|z^{\prime}(s)\right| d s\right)^{q}\left|z^{\prime}(t)\right| d t\right)^{1 / q} \\
= & \left(\int_{a}^{x}\left|f^{\prime}(z(t))\right|^{p}\left|z^{\prime}(t)\right| d t\right)^{1 / p}\left(\frac{\left(\int_{a}^{x}\left|z^{\prime}(s)\right| d s\right)^{q+1}}{q+1}\right)^{1 / q} \\
= & \frac{\left(\int_{a}^{x}\left|z^{\prime}(s)\right| d s\right)^{1+1 / q}}{(q+1)^{1 / q}}\left(\int_{a}^{x}\left|f^{\prime}(z(t))\right|^{p}\left|z^{\prime}(t)\right| d t\right)^{1 / p}
\end{aligned}
$$


and

$$
\begin{aligned}
& \int_{x}^{b}\left|f^{\prime}(z(t))\right|\left|z^{\prime}(t)\right|\left(\int_{t}^{b}\left|z^{\prime}(s)\right| d s\right) d t \\
\leq & \left(\int_{x}^{b}\left|f^{\prime}(z(t))\right|^{p}\left|z^{\prime}(t)\right| d t\right)^{1 / p}\left(\int_{x}^{b}\left(\int_{t}^{b}\left|z^{\prime}(s)\right| d s\right)^{q}\left|z^{\prime}(t)\right| d t\right)^{1 / q} \\
= & \left(\int_{x}^{b}\left|f^{\prime}(z(t))\right|^{p}\left|z^{\prime}(t)\right| d t\right)^{1 / p}\left(\frac{\left(\int_{x}^{b}\left|z^{\prime}(s)\right| d s\right)^{q+1}}{q+1}\right)^{1 / q} \\
= & \frac{\left(\int_{x}^{b}\left|z^{\prime}(s)\right| d s\right)^{1+1 / q}}{(q+1)^{1 / q}}\left(\int_{x}^{b}\left|f^{\prime}(z(t))\right|^{p}\left|z^{\prime}(t)\right| d t\right)^{1 / p}
\end{aligned}
$$

for $x \in(a, b)$. If we add these two inequalities, we get

$$
\begin{aligned}
B(x) & \leq \frac{\left(\int_{a}^{x}\left|z^{\prime}(s)\right| d s\right)^{1+1 / q}}{(q+1)^{1 / q}}\left(\int_{a}^{x}\left|f^{\prime}(z(t))\right|^{p}\left|z^{\prime}(t)\right| d t\right)^{1 / p} \\
& +\frac{\left(\int_{x}^{b}\left|z^{\prime}(s)\right| d s\right)^{1+1 / q}}{(q+1)^{1 / q}}\left(\int_{x}^{b}\left|f^{\prime}(z(t))\right|^{p}\left|z^{\prime}(t)\right| d t\right)^{1 / p} \\
& =\frac{1}{q+1}\left[\ell^{1+1 / q}\left(\gamma_{u, v}\right)\left(\int_{\gamma_{u, v}}\left|f^{\prime}(z)\right|^{p}|d z|\right)^{1 / p}+\ell^{1+1 / q}\left(\gamma_{v, w}\right)\left(\int_{\gamma_{v, w}}\left|f^{\prime}(z)\right|^{p}|d z|\right)^{1 / p}\right]
\end{aligned}
$$

which proves the first inequality in (2.13). We also have

$$
\begin{aligned}
& \ell^{1+1 / q}\left(\gamma_{u, v}\right)\left(\int_{\gamma_{u, v}}\left|f^{\prime}(z)\right|^{p}|d z|\right)^{1 / p}+\ell^{1+1 / q}\left(\gamma_{v, w}\right)\left(\int_{\gamma_{v, w}}\left|f^{\prime}(z)\right|^{p}|d z|\right)^{1 / p} \\
\leq & \max \left\{\ell^{1+1 / q}\left(\gamma_{u, v}\right), \ell^{1+1 / q}\left(\gamma_{v, w}\right)\right\} \\
\times & {\left[\left(\int_{\gamma_{u, v}}\left|f^{\prime}(z)\right|^{p}|d z|\right)^{1 / p}+\left(\int_{\gamma_{v, w}}\left|f^{\prime}(z)\right|^{p}|d z|\right)^{1 / p}\right] } \\
= & {\left[\max \left\{\ell\left(\gamma_{u, v}\right), \ell\left(\gamma_{v, w}\right)\right\}\right]^{1+1 / q}\left[\left(\int_{\gamma_{u, v}}\left|f^{\prime}(z)\right|^{p}|d z|\right)^{1 / p}+\left(\int_{\gamma_{v, w}}\left|f^{\prime}(z)\right|^{p}|d z|\right)^{1 / p}\right] } \\
= & \left.\frac{1}{2^{1+1 / q}}\left[\ell\left(\gamma_{u, w}\right)+\left|\ell\left(\gamma_{u, v}\right)-\ell\left(\gamma_{v, w}\right)\right|\right]^{1+1 / q}\right] \\
\times & {\left[\left(\int_{\gamma_{u, v}}\left|f^{\prime}(z)\right|^{p}|d z|\right)^{1 / p}+\left(\int_{\gamma_{v, w}}\left|f^{\prime}(z)\right|^{p}|d z|\right)^{1 / p}\right] }
\end{aligned}
$$


and

$$
\begin{aligned}
& \ell^{1+1 / q}\left(\gamma_{u, v}\right)\left(\int_{\gamma_{u, v}}\left|f^{\prime}(z)\right|^{p}|d z|\right)^{1 / p}+\ell^{1+1 / q}\left(\gamma_{v, w}\right)\left(\int_{\gamma_{v, w}}\left|f^{\prime}(z)\right|^{p}|d z|\right)^{1 / p} \\
\leq & \max \left\{\left(\int_{\gamma_{u, v}}\left|f^{\prime}(z)\right|^{p}|d z|\right)^{1 / p},\left(\int_{\gamma_{v, w}}\left|f^{\prime}(z)\right|^{p}|d z|\right)^{1 / p}\right\} \\
\times & {\left[\ell^{1+1 / q}\left(\gamma_{u, v}\right)+\ell^{1+1 / q}\left(\gamma_{v, w}\right)\right] } \\
= & {\left[\max \left\{\int_{\gamma_{u, v}}\left|f^{\prime}(z)\right|^{p}|d z|, \int_{\gamma_{v, w}}\left|f^{\prime}(z)\right|^{p}|d z|\right\}\right]^{1 / p} } \\
\times & {\left[\ell^{1+1 / q}\left(\gamma_{u, v}\right)+\ell^{1+1 / q}\left(\gamma_{v, w}\right)\right] } \\
= & \frac{1}{2^{1 / p}}\left[\int_{\gamma_{u, w}}\left|f^{\prime}(z)\right|^{p}|d z|+\left.\left|\int_{\gamma_{u, v}}\right| f^{\prime}(z)\right|^{p}|d z|-\int_{\gamma_{v, w}}\left|f^{\prime}(z)\right|^{p}|d z| \mid\right]^{1 / p} \\
\times & {\left[\ell^{1+1 / q}\left(\gamma_{u, v}\right)+\ell^{1+1 / q}\left(\gamma_{v, w}\right)\right] . }
\end{aligned}
$$

By Hölder's discrete inequality, we have

$$
\begin{aligned}
& \ell^{1+1 / q}\left(\gamma_{u, v}\right)\left(\int_{\gamma_{u, v}}\left|f^{\prime}(z)\right|^{p}|d z|\right)^{1 / p}+\ell^{1+1 / q}\left(\gamma_{v, w}\right)\left(\int_{\gamma_{v, w}}\left|f^{\prime}(z)\right|^{p}|d z|\right)^{1 / p} \\
\leq & {\left[\ell^{q+1}\left(\gamma_{u, v}\right)+\ell^{q+1}\left(\gamma_{v, w}\right)\right]^{1 / q}\left[\int_{\gamma_{u, v}}\left|f^{\prime}(z)\right|^{p}|d z|+\int_{\gamma_{v, w}}\left|f^{\prime}(z)\right|^{p}|d z|\right]^{1 / p} } \\
= & {\left[\ell^{q+1}\left(\gamma_{u, v}\right)+\ell^{q+1}\left(\gamma_{v, w}\right)\right]^{1 / q}\left(\int_{\gamma_{u, w}}\left|f^{\prime}(z)\right|^{p}|d z|\right)^{1 / p}, }
\end{aligned}
$$

which prove the last part of (2.13).

We have:

Corollary 2.2. With the assumption of Theorem 2.4 and if there exists $m \in \gamma$ such that $\ell\left(\gamma_{u, m}\right)=$ $\ell\left(\gamma_{m, w}\right)$, then

$$
\left|f(m) \ell(\gamma)-\int_{\gamma} f(z)\right| d z|| \leq \frac{1}{2^{1+1 / q}(q+1)} \ell^{1+1 / q}\left(\gamma_{u, w}\right)\left[\left\|f^{\prime}\right\|_{\gamma_{u, m} ; p}+\left\|f^{\prime}\right\|_{\gamma_{m, w} ; p}\right]
$$

and if $s \in \gamma$ such that $\int_{\gamma_{u, s}}\left|f^{\prime}(z)\right|^{p}|d z|=\int_{\gamma_{s, w}}\left|f^{\prime}(z)\right|^{p}|d z|$, then

$$
\left|f(s) \ell(\gamma)-\int_{\gamma} f(z)\right| d z|| \leq \frac{1}{2^{1 / p}(q+1)}\left[\ell^{1+1 / q}\left(\gamma_{u, s}\right)+\ell^{1+1 / q}\left(\gamma_{s, w}\right)\right]\left\|f^{\prime}\right\|_{\gamma_{u, w} ; p} .
$$

Finally we have: 
Theorem 2.5. With the assumption of Theorem 2.3, we have

$$
\begin{aligned}
& \quad\left|f(v) \ell(\gamma)-\int_{\gamma} f(z)\right| d z|| \\
& \leq \frac{1}{2}\left[\ell^{2}\left(\gamma_{u, v}\right)\left\|f^{\prime}\right\|_{\gamma_{u, v} ; \infty}+\ell^{2}\left(\gamma_{v, w}\right)\left\|f^{\prime}\right\|_{\gamma_{v, w} ; \infty}\right] \\
& \leq \frac{1}{2}\left\{\begin{array}{l}
\frac{1}{4}\left[\ell\left(\gamma_{u, w}\right)+\left|\ell\left(\gamma_{u, v}\right)-\ell\left(\gamma_{v, w}\right)\right|\right]^{2} \\
\times\left[\left\|f^{\prime}\right\|_{\gamma_{u, v} ; \infty}+\left\|f^{\prime}\right\|_{\gamma_{v, w} ; \infty}\right], \\
{\left[\ell^{2 q}\left(\gamma_{u, v}\right)+\ell^{2 q}\left(\gamma_{v, w}\right)\right]^{1 / q}\left(\left\|f^{\prime}\right\|_{\gamma_{u, v} ; \infty}^{p}+\left\|f^{\prime}\right\|_{\gamma_{v, w} ; \infty}^{p}\right)^{1 / p}} \\
p, q>1 \text { and } \frac{1}{p}+\frac{1}{q}=1, \\
{\left[\ell^{2}\left(\gamma_{u, v}\right)+\ell^{2}\left(\gamma_{v, w}\right)\right]\left\|f^{\prime}\right\|_{\gamma_{u, w} ; \infty} .}
\end{array}\right.
\end{aligned}
$$

Proof. We have

$$
\begin{aligned}
& \int_{a}^{x}\left|f^{\prime}(z(t))\right|\left|z^{\prime}(t)\right|\left(\int_{a}^{t}\left|z^{\prime}(s)\right| d s\right) d t \\
\leq & \max _{t \in[a, x]}\left|f^{\prime}(z(t))\right| \int_{a}^{x}\left(\int_{a}^{t}\left|z^{\prime}(s)\right| d s\right)\left|z^{\prime}(t)\right| d t \\
= & \frac{1}{2} \max _{t \in[a, x]}\left|f^{\prime}(z(t))\right|\left(\int_{a}^{x}\left|z^{\prime}(s)\right| d s\right)^{2}=\frac{1}{2} \ell^{2}\left(\gamma_{u, v}\right)\left\|f^{\prime}\right\|_{\gamma_{u, v} ; \infty}
\end{aligned}
$$

and

$$
\begin{aligned}
& \int_{x}^{b}\left|f^{\prime}(z(t))\right|\left|z^{\prime}(t)\right|\left(\int_{t}^{b}\left|z^{\prime}(s)\right| d s\right) d t \\
\leq & \max _{t \in[x, b]}\left|f^{\prime}(z(t))\right| \int_{x}^{b}\left(\int_{t}^{b}\left|z^{\prime}(s)\right| d s\right)\left|z^{\prime}(t)\right| d t \\
= & \frac{1}{2} \max _{t \in[x, b]}\left|f^{\prime}(z(t))\right|\left(\int_{x}^{b}\left|z^{\prime}(s)\right| d s\right)^{2}=\frac{1}{2} \ell^{2}\left(\gamma_{v, w}\right)\left\|f^{\prime}\right\|_{\gamma_{v, w} ; \infty},
\end{aligned}
$$

which by addition produce

$$
B(x) \leq \frac{1}{2}\left[\ell^{2}\left(\gamma_{u, v}\right)\left\|f^{\prime}\right\|_{\gamma_{u, v} ; \infty}+\ell^{2}\left(\gamma_{v, w}\right)\left\|f^{\prime}\right\|_{\gamma_{v, w} ; \infty}\right]
$$

and by utilising the inequality (2.10), we get the first part of (2.16).

The second part is obvious and we omit the details.

Corollary 2.3. With the assumption of Theorem 2.3 and if there exists $m \in \gamma$ such that $\ell\left(\gamma_{u, m}\right)=$ $\ell\left(\gamma_{m, w}\right)$, then

$$
\begin{aligned}
\left|f(m) \ell(\gamma)-\int_{\gamma} f(z)\right| d z|| & \leq \frac{1}{8}\left[\left\|f^{\prime}\right\|_{\gamma_{u, m} ; \infty}+\left\|f^{\prime}\right\|_{\gamma_{m, w} ; \infty}\right] \ell^{2}\left(\gamma_{u, w}\right) \\
& \leq \frac{1}{4}\left\|f^{\prime}\right\|_{\gamma_{u, w} ; \infty} \ell^{2}\left(\gamma_{u, w}\right) .
\end{aligned}
$$




\section{EXAMPLES FOR CiRCULAR PATHS}

Let $[a, b] \subseteq[0,2 \pi]$ and the circular path $\gamma_{[a, b], R}$ centered in 0 and with radius $R>0$

$$
z(t)=R \exp (i t)=R(\cos t+i \sin t), t \in[a, b] .
$$

If $[a, b]=[0, \pi]$, then we get a half circle, while for $[a, b]=[0,2 \pi]$, we get the full circle. We have

$$
z^{\prime}(t)=R i \exp (i t), t \in[a, b]
$$

and $\left|z^{\prime}(t)\right|=R$ for $t \in[a, b]$ giving that

$$
\ell\left(\gamma_{[a, b], R}\right)=\int_{a}^{b}\left|z^{\prime}(t)\right| d t=R(b-a) .
$$

If $x \in[a, b]$ and $v=R \exp (i x)$, then by (2.8), we have

$$
\begin{aligned}
& \left|R(b-a) f(R \exp (i x))-R \int_{a}^{b} f(R \exp (i t)) d t\right| \\
\leq & R(x-a) R \int_{a}^{x}\left|f^{\prime}(R \exp (i t))\right| d t+R(b-x) R \int_{x}^{b}\left|f^{\prime}(R \exp (i t))\right| d t,
\end{aligned}
$$

that is equivalent to

$$
\begin{aligned}
& \left|(b-a) f(R \exp (i x))-\int_{a}^{b} f(R \exp (i t)) d t\right| \\
& \leq R(x-a) \int_{a}^{x}\left|f^{\prime}(R \exp (i t))\right| d t+R(b-x) \int_{x}^{b}\left|f^{\prime}(R \exp (i t))\right| d t
\end{aligned}
$$

for $x \in[a, b]$. In particular, if we take $x=\frac{a+b}{2}$ in (3.17), then we get

$$
\left|(b-a) f\left(R \exp \left(\frac{a+b}{2} i\right)\right)-\int_{a}^{b} f(R \exp (i t)) d t\right| \leq \frac{1}{2} R(b-a) \int_{a}^{b}\left|f^{\prime}(R \exp (i t))\right| d t .
$$

If $m \in[a, b]$ is such that

$$
\int_{a}^{m}\left|f^{\prime}(R \exp (i t))\right| d t=\int_{m}^{b}\left|f^{\prime}(R \exp (i t))\right| d t,
$$

then from (3.17), we get

$$
\begin{aligned}
& \left|(b-a) f(R \exp (m i))-\int_{a}^{b} f(R \exp (i t)) d t\right| \\
\leq & \frac{1}{2} R(b-a) \int_{a}^{b}\left|f^{\prime}(R \exp (i t))\right| d t .
\end{aligned}
$$

By making use of (2.13), we get

$$
\begin{aligned}
& \left|R(b-a) f(R \exp (i x))-R \int_{a}^{b} f(R \exp (i t)) d t\right| \\
\leq & \frac{1}{q+1}\left[R^{1+1 / q}(x-a)^{1+1 / q} R^{1 / p}\left(\int_{a}^{x}\left|f^{\prime}(R \exp (i t))\right|^{p} d t\right)^{1 / p}\right. \\
+ & \left.R^{1+1 / q}(b-x)^{1+1 / q} R^{1 / p}\left(\int_{x}^{b}\left|f^{\prime}(R \exp (i t))\right|^{p} d t\right)^{1 / p}\right]
\end{aligned}
$$


that is equivalent to

$$
\begin{aligned}
& \left|(b-a) f(R \exp (i x))-\int_{a}^{b} f(R \exp (i t)) d t\right| \\
\leq & \frac{1}{q+1} R\left[(x-a)^{1+1 / q}\left(\int_{a}^{x}\left|f^{\prime}(R \exp (i t))\right|^{p} d t\right)^{1 / p}\right. \\
+ & \left.(b-x)^{1+1 / q}\left(\int_{x}^{b}\left|f^{\prime}(R \exp (i t))\right|^{p} d t\right)^{1 / p}\right]
\end{aligned}
$$

for $x \in[a, b]$. If we take $x=\frac{a+b}{2}$ in (3.20), then we get

$$
\begin{aligned}
& \left|(b-a) f\left(R \exp \left(\frac{a+b}{2} i\right)\right)-\int_{a}^{b} f(R \exp (i t)) d t\right| \\
\leq & \frac{1}{(q+1) 2^{1+1 / q}} R(b-a)^{1+1 / q}\left[\left(\int_{a}^{\frac{a+b}{2}}\left|f^{\prime}(R \exp (i t))\right|^{p} d t\right)^{1 / p}\right. \\
+ & \left.\left(\int_{\frac{a+b}{2}}^{b}\left|f^{\prime}(R \exp (i t))\right|^{p} d t\right)^{1 / p}\right] .
\end{aligned}
$$

If $c \in[a, b]$ is such that

$$
\int_{a}^{c}\left|f^{\prime}(R \exp (i t))\right|^{p} d t=\int_{c}^{b}\left|f^{\prime}(R \exp (i t))\right|^{p} d t
$$

then by (3.20), we get

$$
\begin{aligned}
& \left|(b-a) f(R \exp (i c))-\int_{a}^{b} f(R \exp (i t)) d t\right| \\
\leq & \frac{1}{(q+1) 2^{1 / p}} R\left[(c-a)^{1+1 / q}+(b-c)^{1+1 / q}\right]\left(\int_{a}^{b}\left|f^{\prime}(R \exp (i t))\right|^{p} d t\right)^{1 / p} .
\end{aligned}
$$

Further, if we use (2.16), then we have

$$
\begin{aligned}
& \left|R(b-a) f(R \exp (i x))-R \int_{a}^{b} f(R \exp (i t)) d t\right| \\
\leq & \frac{1}{2} R^{2}\left[(x-a)^{2} \sup _{t \in[a, x]}\left|f^{\prime}(R \exp (i t))\right|+(b-x)^{2} \sup _{t \in[x, b]}\left|f^{\prime}(R \exp (i t))\right|\right] \\
\leq & \frac{1}{2} R^{2}\left[(x-a)^{2}+(b-x)^{2}\right] \sup _{t \in[a, b]}\left|f^{\prime}(R \exp (i t))\right|,
\end{aligned}
$$


that is equivalent to

$$
\begin{aligned}
& \left|(b-a) f(R \exp (x i))-\int_{a}^{b} f(R \exp (i t)) d t\right| \\
\leq & \frac{1}{2} R\left[(x-a)^{2} \sup _{t \in[a, x]}\left|f^{\prime}(R \exp (i t))\right|+(b-x)^{2} \sup _{t \in[x, b]}\left|f^{\prime}(R \exp (i t))\right|\right] \\
\leq & R\left[\frac{1}{4}(b-a)^{2}+\left(x-\frac{a+b}{2}\right)^{2}\right] \sup _{t \in[a, b]}\left|f^{\prime}(R \exp (i t))\right|
\end{aligned}
$$

for $x \in[a, b]$. In particular, we have

$$
\begin{aligned}
& \left|(b-a) f\left(R \exp \left(\frac{a+b}{2} i\right)\right)-\int_{a}^{b} f(R \exp (i t)) d t\right| \\
\leq & \frac{1}{8} R(b-a)^{2}\left[\sup _{t \in\left[a, \frac{a+b}{2}\right]}\left|f^{\prime}(R \exp (i t))\right|+\sup _{t \in\left[\frac{a+b}{2}, b\right]}\left|f^{\prime}(R \exp (i t))\right|\right] \\
\leq & \frac{1}{4} R(b-a)^{2} \sup _{t \in[a, b]}\left|f^{\prime}(R \exp (i t))\right| .
\end{aligned}
$$

We give now examples for some fundamental complex functions. Consider the function $f(z)=z^{n}, z \in \mathbb{C}$ with $n \geq 1$. Then $f^{\prime}(z)=n z^{n-1}$,

$$
\begin{gathered}
f(R \exp (i x))=R^{n} \exp (n x i), \\
\left|f^{\prime}(R \exp (i t))\right|=n R^{n-1}|\exp ((n-1) i t)|=n R^{n-1}, t \in[a, b]
\end{gathered}
$$

and

$$
\int_{a}^{b} f(R \exp (i t)) d t=R^{n} \int_{a}^{b} \exp (n t i) d t=R^{n} \frac{\exp (n b i)-\exp (n a i)}{n i} .
$$

Making use of the inequality (3.23), we get

$$
\left|(b-a) R^{n} \exp (n x i)-R^{n} \frac{\exp (n b i)-\exp (n a i)}{n i}\right| \leq R\left[\frac{1}{4}(b-a)^{2}+\left(x-\frac{a+b}{2}\right)^{2}\right] n R^{n-1},
$$

which is equivalent to

$$
\left|(b-a) \exp (n x i)-\frac{\exp (n b i)-\exp (n a i)}{n i}\right| \leq n\left[\frac{1}{2}(b-a)^{2}+\left(x-\frac{a+b}{2}\right)^{2}\right]
$$

for $x \in[a, b]$. If we take in (3.25) $x=\frac{a+b}{2}$, then we get

$$
\left|(b-a) \exp \left(n\left(\frac{a+b}{2}\right) i\right)-\frac{\exp (n b i)-\exp (n a i)}{n i}\right| \leq \frac{1}{4} n(b-a)^{2}
$$

for $n \geq 1$. Consider the exponential function $f(z)=\exp (z), z \in \mathbb{C}$. Then $f^{\prime}(z)=\exp (z)$,

$$
\left|f^{\prime}(R \exp (i t))\right|=|\exp (R(\cos t+i \sin t))|=\exp (R \cos t), t \in[a, b]
$$


and by the inequality (3.17), we get

$$
\begin{aligned}
& \left|(b-a) \exp (R \exp (i x))-\int_{a}^{b} \exp (R \exp (i t)) d t\right| \\
\leq & R\left[(x-a) \int_{a}^{x} \exp (R \cos t) d t+(b-x) \int_{x}^{b} \exp (R \cos t) d t\right],
\end{aligned}
$$

while from the inequality (3.23), we get

$$
\begin{aligned}
& \left|(b-a) \exp (R \exp (i x))-\int_{a}^{b} \exp (R \exp (i t)) d t\right| \\
\leq & \frac{1}{2} R\left[(x-a)^{2} \sup _{t \in[a, x]} \exp (R \cos t)+(b-x)^{2} \sup _{t \in[x, b]} \exp (R \cos t)\right] \\
\leq & R\left[\frac{1}{4}(b-a)^{2}+\left(x-\frac{a+b}{2}\right)^{2}\right] \sup _{t \in[a, b]} \exp (R \cos t)
\end{aligned}
$$

for $x \in[a, b]$. From the inequality (3.20), we get

$$
\begin{aligned}
& \left|(b-a) \exp (R \exp (i x))-\int_{a}^{b} \exp (R \exp (i t)) d t\right| \\
\leq & \frac{1}{q+1} R\left[(x-a)^{1+1 / q}\left(\int_{a}^{x} \exp (p R \cos t) d t\right)^{1 / p}\right. \\
+ & \left.(b-x)^{1+1 / q}\left(\int_{x}^{b} \exp (p R \cos t) d t\right)^{1 / p}\right]
\end{aligned}
$$

for $x \in[a, b]$, where $p, q>1$ with $\frac{1}{p}+\frac{1}{q}=1$. If in the inequality (3.27) we take $x=\frac{a+b}{2}$, then we get

$$
\begin{aligned}
& \left|(b-a) \exp \left(R \exp \left(\frac{a+b}{2} i\right)\right)-\int_{a}^{b} \exp (R \exp (i t)) d t\right| \\
\leq & \frac{1}{2} R(b-a) \int_{a}^{b} \exp (R \cos t) d t,
\end{aligned}
$$

while from the inequality (2.15), we get

$$
\begin{aligned}
& \left|(b-a) \exp \left(R \exp \left(\frac{a+b}{2} i\right)\right)-\int_{a}^{b} \exp (R \exp (i t)) d t\right| \\
\leq & \frac{1}{8}(b-a)^{2} R\left[\sup _{t \in\left[a, \frac{a+b}{2}\right]} \exp (R \cos t)+\sup _{t \in\left[\frac{a+b}{2}, b\right]} \exp (R \cos t)\right] \\
\leq & \frac{1}{4} R(b-a)^{2} \sup _{t \in[a, b]} \exp (R \cos t) .
\end{aligned}
$$


From (3.29), we have

$$
\begin{aligned}
& \left|(b-a) \exp \left(R \exp \left(\frac{a+b}{2} i\right)\right)-\int_{a}^{b} \exp (R \exp (i t)) d t\right| \\
\leq & \frac{1}{(q+1) 2^{1+1 / q}} R(b-a)^{1+1 / q} \\
\times & {\left[\left(\int_{a}^{\frac{a+b}{2}} \exp (p R \cos t) d t\right)^{1 / p}+\left(\int_{\frac{a+b}{2}}^{b} \exp (p R \cos t) d t\right)^{1 / p}\right] . }
\end{aligned}
$$

Acknowledgement. The author would like to thank the anonymous referees for their valuable comments that have been implemented in the final version of the manuscript.

\section{REFERENCES}

[1] S. S. Dragomir: A refinement of Ostrowski's inequality for absolutely continuous functions whose derivatives belong to $L_{\infty}$ and applications. Libertas Math. 22 (2002), 49-63.

[2] S. S. Dragomir: A refinement of Ostrowski's inequality for absolutely continuous functions and applications. Acta Math. Vietnam 27 (2002), no. 2, 203-217.

[3] S. S. Dragomir: A functional generalization of Ostrowski inequality via Montgomery identity. Acta Math. Univ. Comenian. (N.S.) 84 (2015), no. 1, 63-78. Preprint RGMIA Res. Rep. Coll. 16 (2013), Art. 65, pp. 15 [Online http://rgmia.org/papers/v16/v16a65.pdf].

[4] S. S. Dragomir: Ostrowski type inequalities for Lebesgue integral: a survey of recent results. Aust. J. Math. Anal. Appl. 14 (2017), no. 1, Art. 1, 283 pp.

[5] S. S. Dragomir: An extension of Ostrowski's inequality to the complex integral. Preprint RGMIA Res. Rep. Coll. 18 (2018), Art. 112, 17 pp. [Online https://rgmia.org/papers/v21/v21a112.pdf] .

[6] S. S. Dragomir, S. Wang: Applications of Ostrowski's inequality to the estimation of error bounds for some special means and for some numerical quadrature rules. Appl. Math. Lett. 11 (1) (1998), 105-109.

[7] D. S. Mitrinović, J. E. Pečarić and A. M. Fink: Inequalities for Functions and Their Integrals and Derivatives. Kluwer Academic Publishers, Dordrecht, 1994.

[8] A. Ostrowski: Über die Absolutabweichung einer differentiierbaren Funktion von ihrem Integralmittelwert. Comment. Math. Helv. 10 (1938), 226-227.

\section{Silvestru SEVER Dragomir}

VICTORIA UNIVERSITY

MATHematics, College of ENGINEERING \& SCIENCE

Melbourne City, MC 8001, Australia

UNIVERSITY OF THE WITWATERSRAND

SCHOOL OF COMPUTER SCIENCE \& APPlied Mathematics

Private Bag 3, Johannesburg 2050, SOUth Africa

ORCID: 0000-0003-2902-6805

Email address: sever.dragomirevu.edu.au 